\title{
Changing the Face of Modern Medicine: Stem Cell and Gene Therapy Abstract Author Index
}

Aarrass, H, P435

Abadie, A, P406

Abdelhay, E, P074

Abdo, L M, P127

Abdullin, T, P151

Abdullin, T I, P003, P014

Abken, H, OR001, INV049

Ablinger, M, P292

Abodollahi, A, P062

Abraham, E, P323

Abramov, S A, P050, P051

Abramov, S N, P048

Abramova, Z I, P085, P326

Abyzova, M S, P367

Ackermann, M, P117

Acquati, S, INV002, P053, P332

Adachi, K, P178

Adair, J E, P244

Adjali, O, P386, P412, P488, OR014

Adriouch, S, P474

Aebischer, J, P180

Aebischer, P, P272

Aeby, L, P270

Aerts-Kaya, F, P030, P238

Afione, S, INV043

Afzal, S, P049, P442

Agatieva, E, P452

Agbandje-McKenna, M, INV064

Aghazadeh, M, P206

Agliullina, D R, P243, P390

Ahmad, A, P135

Ahmed, S G, P199, P380

Ahmed, S S, P344

Ahn, S Y, P084

Ahsan, F, P489

Aichele, P, P167

Aimaletdinov, A M, P148, P480

Aiuti, A, INV002, P028, P032, P053, P104, P170, P332

Akcakaya, P, INV069

Akhatova, F S, P012, P013, P168

Akhmetzyanova, E R, P218

Akinci, E, P150

Akyüz, L, OR027

Al-Basti, H B, P121

Al-Khulaifi, M J, P121

Al-Mohannadi, A K, P044

Al-Sulaiti, A M, P121
Al-Zaidy, S, OR005

Albano, L, P254, P261

Alber, A B, P360, P425

Alberquilla, O, P027, P031, P120, P284

Albert, S, P181

Albertini, P, INV002

Albright, C, P251

Aldabe, R, P233

Aldamiz-Echevarria, L, P110

Alexander, I E, P135, P448, OR019

Alexandrushkina, N A, P114,

P118, P407

Alexsson, A, P048

Alfano, L N, OR005

Alfonso, E, INV002

Ali Mohammadi Nafchi, N, P199

Ali, R R, P219, P245

Aliev, R O, P391

Allais, M, P386

Allegri, G, P353

Allen, C, P301

Allen, K L, P258, P349, OR022

Allievi, L, P457

Allouche, J, P164

Almarza, E, P033, P106, OR024

Almeida, M I, P210, P236

Aloia, L, P492

Altunbas, H A, P356

Alvarez, L, P260, OR012

Álvarez, L, P043

Alves, S, INV035

Alzubi, J, OR001

Amaya, A K, OR019

Ambrosi, G, P492

Amendola, M, P042

Amini, L, OR027

Anagnostopoulos, A, P024, P034, P428

Anakok, O F, P290

Andari, J E, P484

Andersson, G, P048

André-Schmutz, I, INV077

Andres-Mateos, E, P337, P491

Andrews, P, INV042

Andrysiak, K, P382

Anegon, I, P113

Anelli, M, INV003

Anggakusuma, A, P463
Anguela, X M, P345

Annoni, A, P254

Antepowicz, A, P308

Antipin, I, P362, P363, P364, P368

Antoine, R, P477

Antonarelli, G, P315

Antoniou, M N, P250

Apicco, D J, P198

Aprile, A, P028

Aragão-Filho, W C, OR020

Aranda, P, P142

Arandel, L, P242, P379

Arbuthnot, P, P441

Arcuri, L, P213

Aristorena, M, P219

Armant, M, P340

Armengaud, J, P435

Armenteros-Monterroso, E, P036

Armour, S M, P345

Arnold, W D, OR005

Arnoldi, F, P262

Aronson, S J, P351, P357

Arora, S K, P077

Arosio, D, P277

Arsenijevic, Y, P214, P215, P234

Arumugam, S, P450

Aryee, M, INV069

Asai, Y, P272

Aslanidi, G, P444

Asperti, C, P314

Asplund, A, P406

Asrih, M, P205

Astord, S, P275

Atochin, D N, P169

Atochina-Vasserman, E N, P155, P169

Attanasio, V, P332

Aubin, J, P178, P185, P190

Aubourg, P, INV076

Auricchio, A, P181, P182, P255, P265, OR021, INV088

Ausseil, J, INV076

Autio, M I, P289

Avanzini, M A, P028

Avgerinou, A, P269, P285

Ayer, J, P444

Ayme, G, P054

Ayuso, E, P348, P488, OR014, INV016, INV066 
Azevedo, P L, P074

Azibani, F, P379

Babarit, C, P109, P348

Baccega, T, INV069

Bachi, A, P229

Baciu, P, P251

Badakul, G, P150

Bae, H, OR017

Baert, M, OR011

Baghban, H, P206

Baila, S, P482

Baines, D, P269

Baker, K, P486

Baker, S, P436

Bakker, R S, P357

Baklaushev, V P, P202

Bal, A, P077

Balci, M K, P295, P347, P356

Baldeschi, C, P164

Baldwin, K, INV060

Ballatore, P, P053

Baltin, M E, P151

Baltina, T, P151

Baltina, T V, P159

Bange, F, P166

Bankiewicz, K S, P438

Bao, G, P244

Baoutina, A, P402

Baptista, R P, P420

Baradez, M O, P313, P420

Barata, J, P101, P183

Barbarossa, L, P315

Barber Janer, A, P443

Barbiera, G, P310

Barbon, E, P054

Barbosa, M A, P210, P236

Bardet, C, P417

Bargsten, K, OR016

Baricordi, C, P339

Barkats, M, P275

Barker, R A, P201

Bart, C I, INV056

Barteneva, N S, P143

Bartholomae, C, P319

Bartoe, J T, P221

Bartolaccini, S, P492

Bartz-Schmidt, K U, OR006

Barzaghi, F, P032, P170

Barzel, A, P298, P336

Basalova, N A, P137, P145, P371

Bascuas Castillo, T, P216

Bascuas, T, P142, P205

Bashirov, F V, P139, P208

Basso-Ricci, L, P028, P032, P053, P104, P170

Bastille, A, P346

Batasheva, S N, P015, P018

Bauer, J W, P231, P263

Bauer, T, P463

Baumann, U, P351

Baumgärtner, W, P166

Bazo, A, P462

Becker, R, OR028
Beissert, T, OR028

Bek, J, P138, P204

Beléndez, C, P027

Belguise, P, P327, P479

Bellec, J, P389

Bellin, M, P355

Bellintani, F, P457

Ben M'Barek, K, P111

Ben Nasr, M, P339

Benabdellah, K, P300

Benati, D, P265

Bendana, Y, P267

Bender, R R, P107

Bendik, M, P047

Benedicenti, F, P332, OR008

Benfenati, F, P193

Benihoud, K, INV015

Benkhelifa-Ziyyat, S, P379, P415

Bensalah, M, P242

Bentzinger, C, P008

Beretta, S, P103, P261

Berghuis, D, OR011

Bergmann, T, P115

Berk, C, OR016

Bernadin, O, P300

Bernardo, M, P361

Bernardo, M E, P028, P032, P053

Berry, K, OR005

Berta, G N, P171

Bertin, B, P001

Bertrand, A T, P379

Beryozkin, A, P214

Besir, H, P413, P416

Besse, A, P275

Beuers, U, P351, P357

Beuvin, M, P379

Bhagwan, J R, P226

Bhat, S, P402

Bhudia, N, P144

Bialek, C, P463

Bianchi, A, P299

Biasco, L, P104, P339

Biavasco, R, P025

Bickes, M, P117

Bido, S, P179, P193, P213, P396

Biel, M, P239, OR006

Biferi, M G, P275

Biffi, A, P332, P339, P340

Biffi, M, P492

Bifsha, P, P141

Bigger, B, P340, OR023

Bigot, A, P242

Biktagirova, E M, P050, P051

Billig, S, P166

Bilyalov, A I, P078, P087, P091

bin Amin, T, P289

Binato, R, P074

Bingen, B O, INV056

Binley, K, P201

Birocchi, F, P066

Bitoun, M, P379

Bjerkvig, R, P068

Bjursell, M, INV069

Blø, M, P068
Blakslee, D, P009

Blank, P, P166

Blanquart, C, P109

Blelloch, R, INV051

Blits, B, P187, P197

Bloemendaal, L, P447

Bloom, K, P167

Blouin, V, P386, P488, OR014

Bobbin, M L, INV069

Boeker, K O, P459

Boelens, J J, P320

Bogliolo, M, P260

Bogoslovskaya, E V, P281

Bohler, S, P108

Bohlooly-Y, M, INV069

Bohuslavova, B, P187

Boisgerault, F, P343

Boitano, T, P228

Boiti, A, P436

Bonafont, J, P302

Bonamino, M H, P127

Bondanza, A, P025

Bonfanti, P, P269

Bonig, H, P026

Bonini, C, P271, P311, INV004

Bonnamy, B, P477

Bonne, G, P379

Bönnemann, C G, P378

Boomkamp, S, P217

Booth, C, P036

Borderon, N, P412

Bordignon, C, P314, OR004

Börner, K, P490

Borroni, E, P487

Borsotti, C, P029, P044, P115, P171

Bortolussi, G, P298, P343, P447

Bosma, B, P454, OR010

Bosma, P J, P351, P357, P447

Bouillé, P, P264

Boulis, N M, P185

Bounoutas, G, P251

Bourdon, A, P386

Bouzelha, M, OR014

Bowerman, M, P196

Boyd, R F, P221

Boye, S E, P221

Boye, S L, P221

Bozo, I Y, P367

Bozza, M, P122, P437

Brás, J P, P210

Braae, A, P486

Brachtl, G, P102

Brady, J, P301

Branciaroli, C, P443, P460

Brandenberg, N, P234

Brantly, M, P384

Braspenning, J, P115

Bravo, J, P210

Bray, A, P443

Bresciani, A, P187

Brigatti, K W, P351

Brigida, I, INV002, P170

Brill, S, OR028

Bringas, J R, P438 
Broccoli, V, P179, P193, P213, P396, OR029

Brody, L, P278

Bron, P, P435

Brouwers, C, P187

Brull, A, P379

Brunet, J F, P005, INV005

Brunetti-Pierri, N, P351

Bruscaggin, V, P487

Brusco, S, P193

Brzezek, K, OR010

Buchberger, A, P135

Buchholz, C J, P107

Buchholz, T, P461, P475

Büchler, M, P122

Buckland, K F, P036

Buengener, C, P047

Buening, H, P442

Bueren, J, P027, OR012, INV026

Bueren, J A, P031, P033, P040, P043, P052, P106, P120, P140, P260, P284, OR024

Buj Bello, A, P376, INV054

Buj-Bello, A, P252

Bulatov, E, P321

Bullen, L, P122

Bullen, L F, P427

Büning, H, OR015, INV013, INV015

Buquicchio, F, P254

Burghes, A HM, OR005

Burilov, V, P362, P363

Burkhardt, L M, OR027

Burns, S, OR020

Buron, J, P191

Burrone, O R, P262

Buscail, L, P061, P070

Bushman, F D, P324

Busser, B, P317

Butler-Browne, G, P242

Buttery, P C, P201

Buzanska, L, P011

Cabanes-Creus, M, P448

Cagin, U, P341, P345

Cai, H, P468

Caiazzo, M, P237

Calabria, A, P041, P332, OR008

Calaminus, C, P256

Calbi, V, P032, P053

Caldana, E, P105, P157

Calin, G A, P236

Calne, R, P235

Camarena, J, P284

Camisa, B, P025

Campbell, A C, P198

Campbell, S L, P354

Campo-Fernandez, B, P023

Camps, G, P233

Canales, M, P246

Canepari, C, P492

Cantore, A, P492, OR031

Capo, V, P254

Cappella, M, P275

Cappellari, O, P375, P392
Capsali, C, P024, P034

Cardinal, J J, P451

Carlon, M, P277

Carmen, J, P301

Carmo, M, P414

Carneiro, M S, P127

Carnevale, E, P311

Carrascoso-Rubio, C, P043, P052

Carrascoso, C, P106

Carreras, A, INV069

Carretero, M, P302

Carrier, L, P226

Carrigan, M, P217

Carriglio, N, INV002

Carrillo, R R, P244

Carroll, J, P185, P190

Carter, T, P178, P190

Cartier, N, INV024, INV035, INV076

Carvalho, L R, P127

Casado-Medrano, V, P309

Casado, J A, P040, OR012

Casini, A, P262, P277, P299

Casiraghi, M, P032

Castagnaro, L, INV002

Castaman, G, P026

Casteels, T, P119

Castelhano, J, P101, P211, P222

Castelli, A, P105, P157

Castelo-Branco, M, P101, P211, P222

Castiello, M C, P254

Castoldi, V, P193

Castro, L, P273

Casucci, M, P314

Catalá, A, OR012

Catala', P, P237

Cathomen, T, P108, P167, P250, OR001, OR002, INV067

Cavazza, A, P268, P283

Cavazzana, M, P042, INV057

Cawood, R, P443, P466, P483

Ceballos, C, P444

Cecchele, A, P229

Cecchini, S, P002

Cecere, F, INV002

Ceiler, J, P442

Centrulo, M, P181

Cereseto, A, P262, P277, P299

Cerullo, V, P073, INV019

Cesana, D, P025, P041, P332, OR008

Chacón-Solano, E, P302

Chadderton, N, P217

Chaikaewkaow, D, P129

Chalothorn, D, OR018

Chalumeau, A, P042

Champion, K M, INV001

Chan, Y H, P401

Chan, Y K, INV086

Chanas, S A, P473

Chandler, R, P491

Chandler, R J, P331, P336, P337, INV043

Chang, C Y, P093

Chang, D, P163

Chang, D Y, P072
Chang, H, P158

Chang, J, P384

Chang, N, P214

Chang, Y, INV068

Chang, Y J, P282

Chao, H, P251

Chapin, J, P037, P038

Charles, S, P054, P343, P383, P485

Charleston, J S, P373

Charpentier, F, P113

Charrier, M, P109

Chassagne, J, P415

Chau, N, P336, P346

Chauzova, T, P112

Chebochakova, A T, P377

Cheeran, M, P165

Chen, F, P185, P190

Chen, J, P228

Chen, Q, P185

Chen, Y, P198

Chen, Y C, P398

Chen, Y H, P436

Cheng, Y F, P401

Cherednichenko, Y V, P015

Chernova, O N, P377

Cheung, W D, P464

Chiang, K, P346

Chicaybam, L, P127

Chien, Y, OR030

Chikunova, E, P420

Chillon, M, P472

Chilton, J, P349

Chin, M S, P257

Chingsuwanrote, P, P133

Chiorini, J A, INV043

Chiriboga-Klein, C A, P188

Cho, C, P253

Cho, Y H, P128, P158, P186

Choavaratana, R, P133

Choi, B, OR017

Choi, E Y, INV043

Choi, J, P136

Choi, J S, P186

Choi, K J, P465

Choi, Y J, P076

Choi, Y K, P128

Chopineau, J, P435

Choquette, T, P318

Christen, B, P322

Christensen, E, P178, P185, P190

Christofi, P, P428

Christophe, O, P035

Christophe, O D, P054

Christou, S, P279

Chuah, M K, P274, P287

Chuang, C Y, P100

Chulpanova, D S, P081, P088, P089, P143, P172, P240

Church, K, OR005

Church, T, P193

Ciana, P, P073

Cianciotti, B C, P271, P311

Cicalese, M P, P032, P053, P104, P170

Ciceri, F, P028, P032, P311, P315 
Cilenti, F, P310

Ciron, C, P348

Cittaro, D, OR008

Ciulla, D, P251

Clare, S, P489, OR020

Clark, A, INV084

Clement, K, INV069

Cloutier, M, P409

Cohen-Tannoudji, M, P275

Colamartino, A, P141

Colangelo, D, P171

Colasante, G, P193

Colecchi, L, P182

Colella, P, P341, P345, P388, P485

Collaud, F, P001, P054, P343, P345, P383, P485

Colle, M, P348

Coltella, N, P066

Combal, J P, P462

Conboy, I, P259

Condino-Neto, A, OR020

Condorelli, G, P227

Connolly, A M, P188

Contestabile, A, P200

Conti, A, P103, P142, P205, P261

Conti, L, P262

Conticello, S, OR021

Contreras Lopez, R, P109

Conway, A, P267

cook, J, OR023

Cooke, A, P235

Cooke, M, P228

Cooper, S, P486

Coppens, M, P026

Coppola, M, P032

Coquin, Y, P445

Coradin, T, P387

Corbetta, S, P457

Cordelier, P, P061, P069, P070

Cordes, M, P039, OR011

Cordier, P, P063, P067

Cordova, G, P242

Corn, J, P259

Corna, S, P314

Cornu, T I, P108, P250, OR002

Corrêa, S, P074

Corteling, R, P430

Cosette, J, P376

Cosset, F L, P029, P300

Costa-Verdera, H, P485

Costa, C, P029, P300

Costa, H, P388

Cota, M, P457

Cotta-Ramusino, C, P254

Couce, M L, P110

Coudray, A, P426, OR026

Courtine, G, INV028

Couzinié, C, P412

Covino, C, P492

Crane, A T, P165, P192

Crasto, S, P227

Crawford, T, P188

Cremers, F P M, P181

Crestani, T, P381
Crippa, L, P457

Crippa, S, P028, P361

Cristofori, P, INV002

Croci, S, OR021

Croft, C, P444

Crombez, E, P110

Crompton, E, P380

Crompton, E M, P199

Crosariol, M, P345

Crosta, C, P482

Croxford, J, P352

Croyle, M A, INV085

Cruz, P E, P444

Crystal, R G, INV023, INV076

Cucci, A, P171

Cuccovillo, I, P489

Cuenca, C, P232

Cugno, C, P121

Culha, M, P013

Cunha-Santos, J, P183

Cunningham, S C, OR019

Cusimano, M, P066

Czajka, M, P079

D'Antiga, L, P343, P351

D'Souza, Z, OR023

DaSilva, J A, P257

Dabbous, O, P395, P404, P409, P411

Daga, S, OR021

Dagna, L, P025

Dahlquist, E, P192

Dahlqvist, J, P048

Dal Ferro, M, P227

Damián, C, P033, OR024

Damiani, D, P200

Daminova, A G, P155, P169

Daniele, N, P341, P383, P485

Danilushkina, A, P147

Danilushkina, A A, P013, P018

Danos, O, P415, INV076

Darin, S, P032, P053

Darras, B T, P188

Dastidar, S, P274

Dautzenberg, I JC, P060

Davari, M R, P206

David, A, OR015

Davidson, B, INV038

Day, J W, P188

de Almeida, L P, P183

De Caneva, A, P298

De Franceschi, F, P008

De Iaco, A, OR026

de Jong, O G, P296

de Knegt, R J, P351

De Koning, E, P125

De Matos, K, P197

De Mattia, F, INV002

de Mollerat du Jeu, X, P481

De Sabbata, G, P343

De Simone, M, INV002

de Simone, S, P181

De Sousa, P F, P127

de Vries, A A, INV056

de Vrij, J, P060
DeVita, N, P439, P491

Debyser, Z, P277

Decembrini, S, P234

Deckert, J, P185

Deev, R V, P240, P243, P367, P377, P390

Deglon, N, P270

Déglon, N, P191, P246, INV022

Deheragoda, M, P333

Dekelver, R, P267

Del Rio Espinola, A, P322

Del Rio Nechaevsky, M, P302

Del Rio-Espinola, A, P319, P324

del Rio, P, P486, OR010

Dell'Aquila, F, P181

della Volpe, L, P103

Della volpe, L, P361

Deluz, C, P305, P360, P425

Demichelis, F, P262, P299

den Dunnen, W, INV035

Denard, J, P376

Deng, D, P444

Deniaud, D, OR014

Deniaud, J, P348

Denis, C V, P054

Denning, C, P226

Deola, S, P044

Deplazes, S, P353

Dergilev, K V, P114

DesJardin, B, P192

Desai, S, P384

Desantis, G, P315

Desnick, R J, P338

Déspres, G, INV035

Dettmer, V, P167, OR001

Devaux, M, P412

Dever, D P, P284

Devoisselle, J, P435

Dewhirst, O, P420

Dhanapal, V, P251

Dhanota, N, P077

Dhawan, A, P333

Dhiab, J, P242

Di Berardino, C, P193

Di Giorgio, A, P351

Di Micco, R, P025, P053, P103, P361

di Micco, R, P261

Di Pasquale, E, P227

Di Pasquale, G, INV043

di Scipio, F, P171

Di Serio, C, P332

Diaz de Heredia, C, OR012

Diaz, G A, P110

Dickson, G, P375, P386, P392

Diez, B, P260

Díez-Cabezas, B, P033, OR024

Dillard, V, P278

Dion, N, P009, P478

Dionisio, F, INV002, P104

Disakou, A, P036

Djouad, F, P109

Doglioni, C, P025

Dolinkin, A O, P469

Doll, K, P019 
Dollive, S, P344

Domanska-Janik, K, P011

Domenger, C, P449

Domingues, S, P164

Donat, T, P191, P270

Donati, F, OR021

Dorange, F, P385

Doria, M, OR021

Doty, A, P419

Doudna, J, P259

Dowling, J J, P378

Droege, M, P395, P404, P409, P411

Du Pasquier, R, P246

du Plessis, F, P454, OR010

Du, Z, P258, P349, OR022

Duarte, M S, P127

Duarte, S, P101, P131

Duarte, S P, P183

Dubart-Kupperschmitt, A, P035, P397

Dubreil, L, P348

Duc, J, P080, P426

Duchateau, P, P108, P307, P317

Duchi, R, P182

Duclert, A, P317

Duffy, M, P483

Duijst, S, P357

Dulak, J, P382, P446

Dunajová, L, P466

Dunnebach, E, P320

Duthoit, C, P264

Dutta, D, P323

Dyikanov, D T, P407

Eden, T, P474

Efimenko, A Y, P118, P137, P145, P160, P371

Eggan, K, INV041

Eggenschwiler, R, P124

Ehl, S, P167

Ehlert, E, P454

Eichhoff, A M, P474

Einsele, H, P328

Eksi, Y E, P295

El Kassar, L, P246

El-Kassem, G, P276

Elkins, S, P439, P491

Ellederova, Z, P187

Ellison, S M, P340

Ellsworth, J L, P344

Elnaggar, M, P044

Eloranta, M L, P048

Elshaer, S L, P189

Ely, A, P441

Engels, M C, INV056

Enjalbert, Q, P417

Erbacher, P, P327, P479

Erbar, S, OR028

Erbs, P, P063, P067

Erceg, S, P232

Eremeev, A A, P159, P208

Eremichev, R Y, P145, P407

Eremin, I, P112, P134

Erendor, F, P347

Erickson, S, P352
Erlacher, M, P108

Erwin, J A, INV062

Escamez, M J, P302

Eschenhagen, T, P226

Escher, J C, P351

Escobar, G, P315

Espinoza, S, P200

Estelien, R, OR009

Estiri, B, P046, P206

Estiri, M, P046, P206

Estrella, N L, P373

Estremores, B, P183

Evans, R, OR003

Evans, W, P189

Evens, H, P287

Everett, J, P324

Evers, M, P187

Evers, M J, P296

Ewenstein, B, P037

Fabry, B T, P256

Fadeev, F O, P139, P208

Faggian, G, P227

Faizov, T, P452

Faizrakhmanova, F A, P377

Fakhrullin, R, P147

Fakhrullin, R F, P010, P012, P013, P018, P149, P168

Fakhrullina, G I, P010

Fallah, A, P046, P062

Fallet, S, P330

Famà, R, P487

Fañanas, S, P031

Fañanas-Baquero, S, P284

Fang, J W, P257

Farias, F HG, P048

Farjadfar, A, P046, P062, P206

Farrar, G J, P217

Farzeneh, F, P340

Fastenrath, M, P420

Fatikhova, G, P363

Faulkner, D, P344

Faust, N, P463

Federici, T, P185

Fedianin, A O, P159

Fehse, B, P068, P456

Feige, J, P008

Felix-Ortiz, A, P178

Fellmann, C, P280

Fellows, E, P192

Fellows, M D, INV069

Feltner, D, P188, P395, P404

Fenelon, G, P201

Feng, Y H, P064

Ferdel, L, P385

Fernández-García, M, P033, OR024

Fernández-Varas, B, P052

Fernandez Muñoz, B, P177

Fernandez-Garcia, M, P106, P140

Fernandez, C A, P257

Fernando, M, P245

Ferrand, M, P445

Ferrari, D, P177

Ferrari, G, INV002, P028, P119
Ferrari, S, P261

Ferreira, A, P191

Ferrer, V, P462

Ferrua, F, P032, P053, P104

Fessart, D, INV021

Ficara, F, P157

Fiedler, C, P047

Figiel-Dabrowska, A, P011

Fillat, C, P334

Findeli, A, P063, P067

Fingerhut, R, P353

Finkel, R S, P188

Finn, J D, OR018

Fiorina, P, P339

Firgens, M, P161

Firth, M A, INV069

Fischer, M D, OR006

FitzPatrick, L M, P286

Flagg, T, P384

Fliegauf, M, OR002

Foale, R, P235

Fodale, V, P187

Follenzi, A, P029, P044, P115, P171, P487

Foloppe, J, P063, P067

Foo, R SY, P289

Forbes, S, INV036

Ford, K, OR018

Forget, A, OR018

Forni, A, P227

Forsayeth, J, P438

forte, G, OR023

Fortin, T, P417

Foust, K D, OR005

Fox, M D, P351

François, V, P386

Franceschini, G M, P262

Francisco, S, P009

Francone, O L, P344

Fraschini, G, P361

Fraser, R, OR010

Fraser, R M, P486

Freund, G, P479

Friman, E T, P305, P360, P425

Fritscher, E, P045

Froelich, C A, P403, P405

Froment, G, P300

Fronza, R, P049, P319, P442

$\mathrm{Fu}, \mathrm{Y}, \mathrm{P} 274$

Fullwood, M J, OR008

Fumagalli, F, P332

Furling, D, P252, P274

Fusco, C, P222

Gálvez, E, P027

Gabaldo, M, INV011

Gabashvili, A N, P202

Gabbasov, R T, P085

Gaffney, D, P226

Gagliardi, G, P194

Gagnon, P, P007, P156, P410

Galibert, L, P453, P471

Galieva, L R, P218

Gallego-Villar, L, P350

Galli, C, P182 
Gallina, P, P025, P041, OR008

Galvez, E, OR012

Galy, A, P445, OR012

Gamba-Vitalo, C, P190

Gamm, D, P234

Gan, L, P373

Gan, S U, P235

Gangadharan, B, P037

Gangaraju, R, P189

Gao, J, P037

Garanina, E E, P152, P207, P218, P476

García-Bravo, M, P293

García-Torralba, A, P293

García-Villoria, J, P334

García, M, P302

Garcia Perez, L, P039

Garcia de Andoin, N, OR012

Garcia-Bravo, M, P031

Garcia-Perez, L, OR011

Garcia-Torralba, A, P031, P120

Garcia, B A, P354

Garcia, S P, INV069

Garcin, P, P061, P070

Gardeux, V, P305

Gareev, B I, P014

Garipov, I I, P365

Garofalo, M, P073

Gasmi, M, P223

Gaspar, B, P340

Gaspar, H B, OR011

Gastaldo, M, P482

Gatina, D Z, P152, P476

Gattillo, S, P032

Gautron, A S, P108

Gayon, R, P264

Gazizov, I, P452

Gea-Sorlí, S, P334

Geberhiwot, T, P110

Gebhardt, J C, P360

Geiger-Schredelsecker, S, P126

Geiger, J, P108

Gelfman, C, P223

Genovese, P, P103, P254, P261, P271, P489

Gentilcore, G, P044

Gentner, B, P166, P315, P489, INV058

Gentry, J, P189

Genua, M, P310

Georgakopoulou, A, P024, P034, P428

Gesualdo, C, P181, P182

Geumann, U, P126

Ghosh, S, INV041

Giacca, M, P262, P298

Giannelli, S, INV002, P053, P104, P193, P213, P396

Giannelli, S G, P179

Giannoukos, G, P251

Gicquel, E, P389

Gifford, R, INV064

Gil-Farina, I, P442

Gilazieva, Z E, P172

Gilbert, P, INV053

Gill, D R, P266, P308, P312

Gimenez, Y, P027, OR012
Ginn, S L, OR019

Ginocchio, V, P351

Giordano, A MS, P489

Giroux, B, P275

Gjata, B, P341, P383, P485

Glajch, K E, P198

Glazkova, D V, P281

Gleitz, H, OR023

Gloskowski, S, P251

Gnani, D, P361

Goethe, R, P166

Gohil, S H, OR003

Golde, T E, P444

Gomes, C, P101

Gomez, M J, P341

Gomzikova, M, P018

Gomzikova, M O, P083, P143, P146

Gong, G, OR018

Gonzalez Cordero, A, P245

Gonzalez-Aseguinolaza, G, P233, P273, P462

Gonzalez-Hernandez, T, P197

Goricar, B, P007

Gorkun, A, P134

Gotta, G M, P257

Gougeon, M L, INV076

Gouin, S G, OR014

Goulet, M, P178

Gourdon, G, P252

Goureau, O, P111, P194

Gouvêa Santos, J, P455

Govindan, S, P306, P425

Gow, A J, P155, P169

Grande, A W, P165, P192

Grandolfo, D, P313

Grassl, M, P463

Gray, D H, P288

Greengard, J, P223

Gregg, J, P324

Gregori, S, INV002

Grellier, B, P063

Grewe, B, INV043

Gribble, F, P235

Grigorieva, O A, P137, P145, P160, P371, P407

Grimbacher, B, OR002

Grimm, D, P449, P474, P484, P490, OR007, INV044

Grinakovskaya, O, P112

Grisch-Chan, H, OR016

Grisch-Chan, H M, P350, P353

Grishanin, R N, P223

Gritti, A, P229

Grivel, J C, P044

Große, S, P490

Growe, J, OR018

Gruber, J, P459

Grueso, E, P328

Grygorev, I, P362, P363, P364, P368

Guével, L, P113

Guarnaccia, C, P343

Gubskiy, I L, P202

Gubsky, L V, P202
Guedan, S, P309

Guenechea, G, P043, P052

Guerchet, N, P341, P485

Guerin, A, P409

Guerrini, I, P285

Guerrouahen, B S, P121

Güenechea, G, P106

Gumerova, A A, P365, P366, P369

Güney-Esken, G, P030, P238

Gunkel, M, P490

Günther, C, P126

Guo, J A, INV069

Gürhan, G, P238

Gurruchaga, J M, P201

Gurvich, O L, P132

Guryanov, I D, P149, P168

Gustincich, S, P200

Guthman, R M, P258, OR022

Gutierrez, A, P300

Guy-Duche, A, P488

Ha, J, P186

Ha, T C, P039

Haake, K, P166

Haan, P, P447

Haas, S, P108

Habeler, W, P111

Häberle, J, P350, P353

Hadaczek, P, P438

Haddad, E, P141

Hagarwal, H, P360

Hainzl, S, P292

Hakimova, E I, P010

Hall, J, OR016

Halloin, C, P117

Hallwirth, C V, OR019

Hamilton, J, P324

Hamilton, J A, P319

Hammer, K, P416

Han, J H, P072

Han, S R, P076

Han, Y, P116, P153

Hanauer, J DS, P107

Hanby, H, P345

Hansen, A, P226

Hanson, G J, P373

Hansson-Hamlin, H, P048

Hantraye, P, P201

Hapil, F Z, P086

Hara, T, P105

Harbaran, S, P375, P392

Harbinski, F, P257

Harbottle, R, P122, P427

Harbottle, R P, P437

Hardcastle, A J, P217

Hardet, R, P388

Harding, C O, P110

Harmening, N, P142, P205, P216, P468

Harmer, S C, P226

Harrasser, M, OR003

Harris, A, OR018

Hart, S, P269, P285

Hartfort, S, OR018

Hartigan, A, P228 
Hartjes, T A, P060

Hartmann, J, OR007

Harvey, R, P286

Haskett, S, P251

Hassan, M, P226

Haurigot, V, P345

Hausser, I, P302

Haworth, K G, P228

Heard, J M, INV076

Heckmann, M B, OR007

Hedlund, E, INV030

Hegde, N, P008

Hein, K, P463

Hein, M, P009, P478

Hempel, T, OR028

Hemsley, K, P342

Henriksen, K, OR025

Henriques, D, P131

Herder, V, P166

Hermann, F G, P126

Hermsen, H, P408, P422

Hernandez Bort, J, P045, P047

Hernandez, R, P028

Hernandez, R J, INV002, P170

Hernando-Llorente, R, P414

Hernando-Rodriguez, M, P106, P140

Herrmann, A, P490

Hersch, S, P190

Hervas, M R, P106, P140

Hetzel, M, P124, P166

Hickey, R D, P258, P349, OR022

Higuchi, T, P335

Hildebrant, E, INV064

Hildenbeutel, M, P108, OR002

Hillin, L G, OR022

Hinojo, I, OR010

Hirsch, D, P126

Hirst, W D, P198

Hishikawa, S, P241

Hivonnait, A, P113

Hladun, R, OR012

Hochmann, S, P102

Hochreiter, A, P102

Hocquemiller, M, P342, INV076

Hodgson, C, P122

Hoehnel, S, P234

Hoffmann, D B, P459

Hoke, J, P219

Holden, K, P294

holley, R, OR023

Hollis, R P, P023

Holmes, M C, P267, P338

Holmes, N, P235

Holt, J R, P272

Hong, Y B, OR017

Hopkins, B, P251

Hoshiya, H, P119

Hossain, J A, P068

Hossbach, M, P185

Hou, J, P178, P185, P190

Howard, K, P201

Howe, S, P144

Howe, S J, P414, P436

Hoyng, C B, P181
Hronowski, X, P198

Hsu, C J, P401

$\mathrm{Hu}, \mathrm{C} \mathrm{J}, \mathrm{P} 401$

Huang, C, P185

Huang, H R, OR018

Huang, S C, P398

Huang, S S, P100

Huang, Y S, P064

Hubbard, B, INV043

Hubbard, B T, P331

Hubert, A, P351

Huch, M, P492

Hudecek, M, P328

Hudjetz, B, P463

Huguet, A, P252

Hulliger, E, OR015

Humbert-Claude, M, P197

Humbert, O, P244

Huston, M W, P338

Hwang, H, OR017

Hwang, J, P116

Hwang, W S, P072

Hwu, W, OR030

Hyde, S C, P266, P308, P312

Hyland, K, P352

Hynynen, H, P453, P471

Iaconcig, A, P343

Iannaccone, S T, P188

Iannacone, M, P492

Iannielli, A, OR029

Ibrahimaibo, D, P322

Iché, A, P264

Iglesias, J M, P486, OR010

Ilyinskii, P, P337, P343

Ilyinskii, P O, P439, P491

Imbeault, M, P426

Indrigo, $\mathrm{M}, \mathrm{P} 179$

Inga, A, P299

Iodice, C, P181, P255

Iodice, D, P310

Iorio, R, P351

Iouranova, A, P426

Ironside, C, P228

Isaaki, R, P478

Isaev, A A, P240, P243, P377, P390

Islamov, R R, P139, P208, P452

Issaragrisil, S, P130, P133, P154, P230

Ivanov, A I, P087

Ivics, Z, P216, P328, P468

Izmailov, A A, P139, P208

Izotova, N, P036

Izsvák, Z, P024, P034, P142, P216, P468

Jacob, A, P261

Jacobs, R, P441

Jaderberg, M, P082

Jaenisch, R, OR013, INV090

Jäger, D, P019, P437

Janan, M, P154

Janciauskiene, S, P124

Jangsangthong, W, INV056

Janosz, E, P124

Jans, T, P274
Jansen, J P, P395

Janssen, K A, P354

Jarraya, M, P111

Jaulin, N, P412

Jauze, L, P383

Jayandharan, G, P450

Jayawardena, M, P420

Jazwa-Kusior, A, P446

Jeschke, M, P318

Jez, M, P382

Jha, K, P189

Ji, Y, P481

Jiamvoraphong, N, P130

Jiang, F, P280

Jiang, H, P251

Jinek, M, OR016

Jiraritthamrong, C, P230

Jividen, H, P384

Jo, D, P253

Jo, S, P069

Johansson, C, P308

Johnen, S, P142, P216, P468

Johnson, B, P178, P185

Johnson, S, P436

Jollet, A, P379

Jones, S, P340

Jonkmans, N, P246

Joseph, J V, P068

Joshi, S, P294

Joung, J K, INV069

Joung, K, P274

Jozkowicz, A, P446

Józkowicz, A, P382

Juillerat, A, P307, P317

Juksar, J, P125

Julien, L, P415

June, C H, P309

Jung, F, OR007

Jung, J, P163

Jung, J H, P072

Junge, N, P351

Jürchott, K, P102

Kaba, A, P150

Kabwe, E, P138

Kachel, B, P449

Kaewjunun, C, P133

Kahle, N, OR006

Kailaanmäki, A, P132, P471

Kaiser, R A, P349, OR022

Kaiser, R M, P258

Kajaste-Rudnitski, A, P489

Kaji, K, INV083

Kakava, S, P237

Kaler, S G, INV043

Kaligin, M S, P366, P377

Kalinina, N I, P145

Kalinke, U, P166

Kamalieva, R, P147

Kamalieva, R F, P018

Kamalov, A A, P371

Kamalov, M, P151

Kamik, N, P478

Kampmann, P, P026 
Kaneda, Y, P071, P093

Kang, E, P116, P136, P153

Kang, M, P458

Kania, A, P382

Kao, B, P386

Kao, S C, P392

Kara, B, P436

Karamalikov, S A, P078, P081, P087, P091

Karch, K R, P354

Karpes, L B, P451

Kärnä, R, P453, P471

Kasahara, N, P075

Kaspar, B K, OR005

Kassim, S, P324

Katili, P, P226

Katsarou, A, P316

Katsoupi, P, P486

Katus, H A, OR007

Kay, M A, P298, P336

Kay, R, P235

Kazuki, Y, P119

Kazunori Akimoto, K A, P291

Keating, A, P387

Kedinger, V, P327, P479

Keir, A G, P288

Kekarainen, T, P132

Keller, G, INV082

Kells, A, P190

Kelmemi, K, P351

Kelso, R, P294

Kempf, H, P117

Kempf, J, P063, P067

Kenna, P F, P217

Kennedy, W P, P333

Ketterl, N, P102

Kewes, H, P463

Khafizova, F A, P017

Khaiboullina, S F, P152, P218

Khalaj, M, INV043

Khan, A, P110

Khanahmad, H, P062

Khaziakhmatova, O G, P155

Kheolamai, P, P133, P154, P230

Khlusov, I A, P155

Kiem, H P, P228, P244

Kim, A, P163

Kim, D, P116, P253, OR017

Kim, H, P116, P136

Kim, H J, P186

Kim, H K, OR017

Kim, J, P186, P253, P297, P458

Kim, J H, P076, P399, P465

Kim, K, P297

Kim, K J, P465

Kim, S, P163, OR017

Kim, S.-S, P072

Kim, U, P253

Kim, Y S, P458

Kimura, M, P075

Kip, A, INV056

Kippen, N, P486, OR010

Kirchgaessner, M, P319

Kirpatovsky, V I, P371

Kirsebom, F, P308
Kishimoto, T K, P337, P343, P439, P491

Kiss, S, P223

Kissel, J T, OR005

Kit Tan, T, P312

Kitaeva, K V, P083, P088, P143

Kiyasov, A P, P146, P365, P366, P367, P369, P391

Kizhakayil, D, P044

Klaic, L, P189

Klamroth, R, P026

Klausegger, A, P263, P292

Kleanthous, M, P250, P279

Klein, A, P252

Klein, P, P375

Kleine Holthaus, S, P219

Kleinlogel, S, OR015

Kletukhina, S K, P146

Klincumhom, N, P129

Kloc, M, P245

Kloth, C, P392

Knoblich, J A, INV089

Kobayashi, H, P335

Kocher, J, P191

Kocher, T, P231, P263, P292

Koch-Nolte, F, P474

Koenig, S, OR002

Kohl, S, OR006

Kohn, D B, P023, P288

Kok, Y L, P108

Koller, U, P231, P263, P292

Konstantinova, P, P187

Koo, O J, P282

Koo, T, P297

Kopinski, P K, P354

Korbelin, J, P396

Korchunjit, W, P130

Korotkova, E, P155, P169

Korsakov, I, P112, P134

Kosheleva, N, P134

Koshpaeva, E S, P139

Kosimova, S F, P369

Kostelec, T D, P007, P156, P410

Kostennikov, A A, P207

Kostic, C, P214, P215

Kotin, R, INV064

Kotoneva, S, P471

Koufali, A, P024, P034

Kouvatsi, A, P034

Kovac, M, P391

Kozyrev, S V, P048

Krämer, C, P490

Krakowiak, J, P466

Kraszewska, I, P446

Kraus, B, P047

Kreher, N C, P330

Krieger, J E, P381

Krijnse-Locker, J, P341

Krishna, V D, P165

Krivega, I, P267

Krombholz, F, P108

Kropp, M, P142, P205, P216, P468

Kruger, W, P350

Kruhmann, R, OR007

Kryuchkova, M A, P015
Ksembaev, S, P452

Kubo, S, P075

Kudryavtseva, V, P155

Kuhlendahl, S, P413, P416

Kühle, J, OR001

Kuhn, T, P360

Kühnel, M P, P166

Kuku, G, P013

Kulebyakin, K Y, P145, P407

Kullmann, D M, P193

Kumar, M, P450

Kuntz, N, P378

Kuntz, N L, P188

Kunze, M, P108

Kuo, C Y, P288

Kuo, H C, P100

Küppers-Munther, B, P406

Kuryk, L, P073, P082

Kuzman-Nichols, N, P165

Kuznecova, S V, P014

Kuznetsova, E S, P137, P160

Kuztetsov, M S, P208

Kwak, G, OR017

Kyratsous, C, OR018

L'Italien, J, OR005

Laaksonen, I, P471

Labbrozzi, J P, P009

Labrune, P, P351

Lachmann, N, P117, P166

Lachmann, P J, P135

Lackner, N, P292

Lad, Y, P201, P387

Lafontaine, D L, P027

Laforêt, P, P383

Lagalice, L, P348

Lagauzere, S, P276

Lagutina, I, P182

Lai Kee Him, J, P435

Lai, K, OR018

Lamana, M L, P106, P140, OR012

Lamazière, A, INV035

Lambert, T, P035

Lamfers, M LM, P060

Lamirault, G, P109

Lamouroux, L, P264

Lamppu, D, P344

Langui, D, INV035

Lankester, A, OR011

Lankester, A C, P039

Laowtammathron, C, P133, P154, P230

Laplaud, D A, P246

Lara-Astiaso, D, P273

Larcher, F, P302

Larcher, T, P113, P348

Latif, A, P068

Latournerie, V, P376

Latulippe, D R, P418

Laufer, R, P342

Laufs, S, P049

Lavrov, I A, P159

Lawlor, M W, P333, P378

Lazzara, G, P010

Lazzari, G, P182 
Le Bec, C, P385

Le Duff, J, P412

Le Guiner, C, P386

Le Heron, A, P386

Le Saux, S, P435

Le brun, P R, P383

León, C, P302

Leathers, R, P481

Leblanc, T, P027

Leborgne, C, P001, P343

Lebreton, C, P386

Lech, W, P011

Lecomte, E, P488

Lecomte, Q, P488

Lederer, C W, P250, P279

Ledevin, M, P113

Lee, B H, P116

Lee, B K, P136

Lee, C H, P076, P399

Lee, C M, P244

Lee, H, P186

Lee, H O, P350

Lee, I H, P100

Lee, J, P253, OR017

Lee, J M, OR017

Lee, J Y, P186, OR017

Lee, K, P253, P259, P280

Lee, K I, P398

Lee, K J, OR017

Lee, K O, P235

Lee, M, OR019

Lee, N, OR030

Lee, R, P116

Lee, S, P153

Lee, S H, P186

Lee, S W, P076, P399

Lee, S Y, P128, P158

Lee, Y, P116, P153

Lee, Y H, P282

Lee, Y J, P072

Leebeek, F, P026

Leenstra, S, P060

Legallais, C, P397

Legrand, P, P435

Leinonen, H, P471

Lemaitre, G, P164

Lemieux, W, P141

Lemus-Diaz, N, P459

Lengler, J, P047

Lenin, R, P189

Lenting, P J, P054

Leocani, L, P193

Leon-Rico, D, P036

Leonard, D, P048

Lepetit, H, P201

Leroux, I, P109, P113

Lesch, H, P471

Lesch, H P, P453

Leskovec, M, P007

Lettera, E, P025, P053, P361

Lettner, T, P292

Leuchs, B, P442

Leung, S, P439, P491

Lever, A, P235
Lévy, C, P029

Lew, J, P444

Lewandowski, M H, P382

Li, C, P024

Li, L, P301, P337, P491

Li, N, P313

Li, Y, P478

Li, Z, P464

Liao, J, P336, P346

Liao, S H, OR019

liao, A, OR023

Lichty, B, P418

Lieber, A, P024, P034

Liemberger, B, P231

Lieubeau, B, P109

Lignani, G, P193

Likhite, S, OR005

Lillegard, J B, P258, P349, OR022

Lim, J, P192

Lim, K, P297

Lim, M C, OR008

Lin, J, P110

Lindblad-Toh, K, P048

Lindsay, H, OR016

Lipponen, E, P471

Lis-Slimak, K, P226

Lisjak, M, P298

Lisowski, L, P448, OR019

Litvin, Y A, P391

Litvinov, R I, P155, P169

Litvinova, L S, P155

Liu, J, OR018

Liu, L, P071, P178, P185, P190

Liu, Q, P443, P460, P466

Liu, X, P444

Livesey, R, INV080

Llado-Santaeularia, M, P255

Lo Nigro, A, P125

Lo Presti, V, P320

Lo Rizzo, C, OR021

Lo Scrudato, M, P252

Lockey, T D, P403, P405

Löfvall, H, OR025

Logan, G J, P135

Lombardo, A, P171

Long, J D, P288

Loos, N, P413, P416

Lopergolo, D, OR021

Lopez-Manzaneda, S, P031

Lopez, F, P061

Lopez, R, OR012

López-Manzaneda, S, P120, P273

Lorain, S, P379, P415

Lorant, J, P109

Lorenzetti, F, P457

Lorenzetti, F C, OR021

Lorenzi, M, P395

Lorenzin, F, P262, P299

Lorthongpanich, C, P130, P133

Lostal, W, P389

Loubière, C, P035

Loucari, C, P250

Loucari, C C, P279

Loveluck, K, P257
Low, W C, P165

Lowes, L P, OR005

Lozano, M L, P052

Lu-Nguyen, N, P375

Lu, W C, P165

Lu, Y C, P401

Lubelski, J, P009, P454, P478, OR010

Luce, E, P035

Ludwig, T, INV092

Lukovic, D, P232

Lulay, C, P442

Lulka, H, P061, P070

Luna, J, P334

Luong, D, P003, P006, P014, P016

Luoni, M, P179

Lupo, M, P181

Lusito, E, P310

Lütke-Eversloh, M, OR027

Lutolf, M, P008, P234

Lux, C, P244

Luz-Crawford, P, P109

Lvova, I D, P159

Maasch, C, P408, P422

MacDonald, I M, INV034

Macown, R, P420

Maeder, M, P251

Maepa, M B, P441

Maestro, S, P233

Maffioletti, S M, P119, P247

Magnani, Z, P271, P311

Magni, S, INV002

Magro, G, P215

Mahajan, S, P060

Mahmoodi, A, P438

Mainot, S, P035

Majumdar, D, P274

Majumder, P, P245

Majzoub, R, OR018

Makani, L, P267

Makarevich, P I, P114, P118, P160, P407, P469

Makarov, E, P363

Makemaharn, O, P133

Malagon-Lopez, J, INV069

Malashchenko, V V, P155

Malatinkova, E, P049

Male, F, P324

Malecki, M, P079

Malerba, A, P375, P386, P392

Malfatti, E, P383

Malinen, J, P471

Malpartida, A B, P237

Managò, F, P200

Mancini, I, P447

Mangeot, P E, P300

Mangiameli, E, P229

Manguán-García, C, P052

Mannhardt, I, P226

Manstein, F, P117

Marais, T, P275

Maranga, L, P002, P467

Marcelo, A, P101, INV035

March, O P, P292 
Marco, E, P251, P257

Marcovecchio, G, P254

Marenna, S, P193

Maresca, M, INV069

Margulies, C, P254

Marie, B, P386

Marie, C, P142, P216, P468

Marigo, V, P265

Marinas, L, P302

Marinello, M, P376

Markosyan, V A, P139, P208

Marktel, S, P028

Marmier, S, P054, P485

Marongiu, M, P436

Marquéz, J, P177

Martellato, D, P482

Marteyn, A, P164

Martin, C, P215

Martin, F, P300

Martin, N, P264

Martin, S, P376

Martinant, C, P164

Martinez-Turrillas, R, P273

Martinez-de-la-Torre, Y, OR004

Martino, S, P229

Maru, B, P395

Mary, B, P450

Masgutov, R, P203, P204

Masgutova, G, P138, P203, P204

Masgutova, G A, P148

Mashinchian, O, P008

Masiuk, K E, P023

Mason, C, P330

Massimino, L, P193

Masson, Y, P164

Mastrobattista, E, P296

Masurier, C, P001

Maswood, R, P219

Matafora, V, P229

Mateu-Bosch, A, P334

Mathias, A, P246

Matos, C, INV035

Matthijssen, L, P060

Maule, G, P262, P277, P299

Maures, T, P294

Maurier, V, P348

Mauro, V, INV002

Maurya, S, P450

Mavilio, F, P333, P378

Mavlikeev, M O, P367, P369, P377

Mayr, L M, INV069

Mayrhofer, J, P047

Mazariegos, G, P333

Mazitova, A M, P085

Mazzucca, D M, P115

McCaroll, S, INV041

McKay, T, P427

McKay, T R, P286

McKiernan, P, P333

McMillan, S, P009, P454, P478, OR010

Mckay, T, P122

Meagher, M M, P403, P405

Mechmet, F, INV035

Medina, M F, P418
Meert, L, P274

Meijer, K, P026

Meireles-Filho, A, P305

Meissner, A, INV072

Mello, C, INV041

Melnikov, P A, P202

Meloni, I, OR021

Mencía, A, P302

Mendell, J R, P188, OR005

Mendonça, L, INV035

Mendonça, L S, P131

Meneghini, V, P042

Menier, M, P404

Menoret, S, P113

Mercuri, E, P254

Merelli, I, P103, P261

Mereu, M, P200

Merienne, N, P246

Merino, E, OR012

Merkelj Koren, J, P007, P410

Merlin, S, P029, P115, P171, P487

Mert, U, P086

Merten, O W, P001

Mesa Infante, V, P197

Mesa-Núñez, C, P033, OR024

Messina, A, P035, P397

Metzner, K J, P108

Meunier, C, P246

Mevel, M, OR014

Meyer, E, P108

Meyer, K, P338, OR005

Mhaidly, R, P029

Miccio, A, P042

Michalakis, S, P239, OR006

Michaud, A, P439, P491

Midroit, M, P205

Miesbach, W, P026

Migliavacca, M, P032, P053, P104

Miglietta, S, INV002

Mikkola, H, INV074

Milani, M, P492

Milani, R, P032

Milardi, G, P170

Miletic, H, P068

Miller, J, P267

Mills, P, P286

Milsom, M, P122

Mingalimova, E Y, P078, P087, P091

Mingozzi, F, P001, P054, P200, P341,

P343, P345, P351, P383, P388, P485

Minopoli, R, P181

Mintaev, R R, P281

Minter, L M, P126

Miragoli, M, P227

Miralles, M, P472

Miranda, C O, P101, P183, P211

Miravet, S, P472

Mirgazizov, R M, P017

Mironova, D, P362, P363

Mirow, M, P456

Miskey, C, P328

Miskin, J E, INV007

Mitchell, J, INV041

Mitrophanous, K, P387
Mitrophanous, K A, P201

Miwa, S, P335

Mizukami, H, P241

Mnyandu, N Z, P441

Moalli, F, P492

Modamio, S, P302

Modrow, S, P444

Moggs, J, P319, P324

Mohamadi, A, P054

Mohammadi Nafchi, N A, P380

Mole, S, P286

Mole, S E, P219

Molinos-Vicente, A, P293

Molinos, A, P031

Møller, A SW, P082

Monahan, P, P038

Monjezi, R, P328

Montagna, C, P262, P277, P299

Montaldo, E, P032, P310

Monteverde, S, INV002

Monti, I, INV002

Monti, L, INV002

Montiel-Equihua, C, P144

Montiel-Equihua, C A, P414

Montini, E, P025, P041, P332, OR008

Monville, C, P111

Moon, D H, P465

Morabito, G, P179, P193, P396

Moran, S, P486

Moreira, R, P131

Morena, F, P229

Morille, M, P435

Moritz, T, P124

Morizane, A, INV073

Moroski-Erkul, C, OR018

Morozova, Y, P364, P368

Morrison, C J, P451

mortazavidehkordi, N, P046, P062, P206

Mortellaro, A, P170

Mosca, F, P053

Moscatelli, I, OR025

Moser, R, P234

Mosqueira, D, P226

Motakis, E, P289

Motlik, J, P187

Mouly, V, P242

Moura, S, P236

Moya-Nilges, M, P341

Moyle, L M, P119

Mrad, R, P351

Mucci, A, P166, P315

Muggeo, S, P157

Mukhamedshina, Y O, P207, P218

Mullakhmetova, A, P138, P203

Müller, N, P413

Müller, O J, P019, OR007

Müller-Felber, W, P378

Mullin, R I, P003

Mummery, C L, P355

Muneer, M, P121

Muntoni, F, P119

Muraine, L, P242

Muramatsu, S, INV087 
Murillas, R, P302

Murlidharan, G, P178

Muro, A, P447

Muro, A F, P298, P343

Murray, B, OR018

Murray, J D, P419

Murthy, N, P259, P280

Mussolino, C, P108, P250, OR002

Mutis, T, P316

Myer, V E, P257

Myers, E B, P435

Nada, A, P311

Naeem, A, P245

Nagendran, S, OR005

Naidoo, J, P438

Nair, N, P287

Najafipoure, S, P062

Nakamaru-Ogiso, E, P354

Nakauchi, H, INV081

Naldini, L, INV002, P066, P103, P254, P261, P271, P315, P332, P489, P492, INV020

Namestnikova, D D, P202

Nan, Z, P165

Naroditskyi, B, P452

Natarajan, P, P301

Nathwani, A, P235

Nathwani, A C, OR003

Naumenko, E A, P018, P148, P149, P168

Navarro, S, P027, P031, P040, P120, OR012

Negre, O, P244, INV027

Negroni, E, P242

Nelson, I, P379

Nemesh, J, INV041

Neri, E, P381

Neri, M, P457

Neukirch, L, P019

Neustroeva, O A, P146

Nevzorova, T A, P169

Newmark, J, P195, P440

Nguyen, A, P166, P223

Nguyen, A H, P117

Nguyen, D, P251

Nguyen, H P, P256

Nguyen, N, P392

Nguyen, N T, INV069

Nguyen, T, P178, P185, P190

Nguyen, T H, P035

Nicastro, E, P343

Nichols, T, P492

Nicolas, C T, P258, P349, OR022

Nicoletti, S, P141

Nierkens, S, P320

Nieto-Romero, V, P293

Nieto, V, P031

Nikitina, M Y, P169

Nilsson, J, P325

Nimiristky, P P, P114

Nimiritsky, P P, P118, P137, P160, P469

Nimmo, R, P387

Ninzima, S, P068
Niopek, D, P449

Niro, A, P179

Nishikawa, T, P071

Nishimura, S, P241

Nist-Lund, C A, P272

Nitsch, R, INV069

Nobles, C, P324

Nobre, R J, P101, P211, P222

Nobrega, C, INV035

Nóbrega, C, P101

Nonnenmacher, M, P185

Norata, R, INV002

Nordin, J, P345

Norelli, M, P025

Noursadeghi, M, P489

Noursalehi, M, P333, P378

Nousiainen, A, P455, P470

Novack, A, P404

Nusco, E, P255

Nyamay'Antu, A, P327, P479

Nyns, E C, INV056

O'Callaghan, C, P269

O'Connell, D, OR018

O'Conner, J, P323

O'Doherty, M, P144

O'Leary, C, OR023

O'Neil, C, P439

O'Riordan, C, P438

Obermayer, A, P123

Odate, S, OR018

Odenthal, M, OR015

Odenwald, C, P413, P416

Ofir, R, P123

Ofrim, M, P269

Ogrinc, F, P188

Ogurcov, S V, P207

Ohashi, T, P335

Ohmori, T, P241

Ohno, K, P438

Ohobotov, D A, P371

Oladimeji, O, P065

Olagüe, C, P273, P462

Olfat, S, P197

Olgasi, C, P115, P171

Oliveira, N, P074

Olivieri, M, P299

Omelchenko, D O, P281

Omelyanenko, N P, P160

Ometti, M, P053, P361

Omrani, M, P104

Ontiveros, M, P472

Ordazzo, G, P179, P213, P396

Orlova, V, P355

Ormoli, L, OR008

Oruetxebarria, I, P471

Oshimura, M, P119

Ostuni, R, P032, P310

Ouared, A, P191

Önder, T, P238

Özçay, F, P351

Özyüncü, Ö, P030

Ovchinsky, N, P333

Ovelgönne, J H, INV047
Ozay, E I, P126

Ozdemir, T, P247

Péréon, Y, P109

Pacouret, S, P488

Pagan, D, P061

Pagani, R, P482

Pagant, S, P338

Page, E, P460

Pahalina, I A, P208

Paiva, V H, P101, P211

Paixao, S, P222

Palchaudhuri, R, P228

Palfi, A, P217

Palfi, S, P201

Pallant, C, P436

Palus-Chramiec, K, P382

Pan, B, P272

Panzner, S, P276

Papa, F T, OR021

Papadimou, E, P237

Papaleo, F, P200

Papasavva, P, P250

Papayanni, P, P428

Papayannopoulou, T, P024

Pappa, V, P237

Pardo, M, P444

Parfyonova, Y V, P114, P469

Parianos, M, P444

Park, B Y, P128, P158

Park, E Y, P128, P158

Park, H, P259, P280

Park, J, P116, P136, P153

Park, K, P128, P158, P186

Park, S, P116, P153, P253

Park, T K, P186

Parker-Manuel, R, P460, P466

Passini, M A, P373

Patrizi, C, P265

Patsali, P, P250, P279

Pattabhi, S, P244

Patzke, H, P185

Paulis, M, P105, P157, P227

Paulusma, C C, P357

Pavasant, P, P129

Pavlou, M, P239

Pavlovic, G, P264

Pavlovski, I, P044

Payne, T, P443, P460, P466

Pearson, R A, P219

Peccate, C, P415

Pechan, P, P190

Pedregal, A, P457

Peking, P, P102

Peljhan, S, P156

Pellin, D, P104

Pells, S C, P430

Peltola, S, P453, P471

Pena, L DM, P188

Penalva, C, P472

Penaud-Budloo, M, P488

Peng, K, INV033

Pennock, S, P195

Pentecost, M, P189 
Penzes, J, INV064

Pereira de Almeida, L, P101, P131, P211, P212, P222, INV035

Pereira, D, P101

Pereira, I, P036

Pereira, U, P397

Perez, A M, P244

Periasamy, R, P189

Perkovic, M, OR028

Perona, R, P052

Perota, A, P182

Perriard, G, P246

Perrier, A, P164

Perrier, A L, P246

Perrin, A, P289

Perriot, S, P246

Perry, C E, P354

Peschanski, M, P111, P164

Peters, M, P352

Peters, T, OR006

Peterson, C W, P244

Petit, S J, P489

Petrella, L, P101, P211, P222

Petrikina, A, P112

Petrillo, C, P489

Petris, G, P262, P277, P299

Petrova, N, P151

Petukhov, A, P321

Peviani, M, P339

Pfeiffer, A, P107

Philip, R, INV008

Philipsen, S, P279

Phornwilardsiri, S, P133

Piétri-Rouxel, F, P415

Pichler, B J, P256

Pichon, C, P067

Pichon, J, P348

Pickett, H A, OR019

Piedra, J, P472

Pielberg, G R, P048

Pietikäinen, S, P132

Pietrasanta, C, P053

Piguet, F, INV076

Pijnappels, D A, INV056

Pike-Overzet, K, P039, OR011

Pinello, L, INV069

Pintado-Berninches, L, P052

Pintauro, R, P187

Pinton, L, P247

Piovani, B, P457

Piras, B A, P403, P405

Piras, F, P489

Pivneva, I, P409

Plaksa, I L, P367

Plancheron, A, P111

Planet, E, P080

Plantinga, M, P320

Plati, T, P492

Plomp, J J, INV056

Poddar, S, P108

Podkovyrina, J, P148

Poelma, R H, INV056

Poelvink, P, P392

Poi, Y, OR010
Poirot, L, P307, P317

Pokrovskaya, L, P155

Poletti, V, P339

Ponce de Leone, V, P234

Pontis, J, OR013

Ponzoni, M, P025

Porcellini, S, P314, OR004

Porritt, M J, INV069

Porro, D M, P482

Porro, F, P298

Porteus, M, P284

Posey Jr, A D, P309

Potenza, A, P271

Potluri, P, P354

Poulard, K, P252

Poupiot, J, P388

Prandi, D, P262, P299

Prasad, S, P333, P378

Prince, N, P197

Prins, H J, P316

Prior, T W, OR005

Priori, S, INV055

Prondzynski, M, P226

Prosper, F, P273

Prudhon, B, P379

Prudnikov, T S, P083, P088, P143

Psatha, N, P024, P428

Puceat, M, INV018

Pugni, L, P053

Pujol, R M, OR012

Pulin, A, P112, P134

Purbhoo, M, P313

Pushkin, R, P384

Puttonen, K A, P132

Puzzo, F, P341, P345, P485

Pythoud, C, P270

Qasim, W, INV032

Qiu, B, P237

Quéméneur, E, P067

Quaranta, P, P032, P053

Queitsch, I, P413, P416

Quemeneur, E, P063

Quintana-Bustamante, O, P031, P120, P284

Quintana, O, P140

Rabienia, M, P206

Raccaud, M, P360

Radeke, R, P267

Radtke, S, P244

Rafiei Hashtchin, A, P117

Ragazzi, M, P119

Rahim, F, P351

Rai, R, P268, P283

Rajan, R, P486, OR010

Rajavel, K, P037, P038

Ramadier, S, P042

Ramalho, A, P277

Ramosaj, M, P270

Rancoita, P, P332

Ranganathan, S, P333

Ranghetti, A, P066

Raninger, A, P123

Rannou, A, P113
Rao, A, P259, P280

Raposo, A, P414

Rapti, K, OR007

Rasponi, M, OR029

Ravassard, P, P477

Rawlings, D J, P244

Raya, A, P171

Razov, A, P416

Rebar, E, P267

Recchia, A, P265

Recino, A, P235

Recordati, C, P105, P157

Redaelli, D, INV002, P053, P332

Reddy, S, P324

Reddy, S S, P244

Reginald, A, P278

Reginato, G, P299

Regio, S, P191, P270

Reichelt, J, P231, P263, P292

Reid, K, P286

Reimann, F, P235

Reinsch, C, P276

Reinke, P, OR027

Reischl, I, INV009

Reiter, M, P410

Relvas, J, P210

Ren, X, P185

Rendina, A, P237

Renieri, A, OR021

Renner, M, INV048

Revkova, V A, P202

Rey, M, P270

Reyon, D, P274

Rhoden-Smith, A, OR018

Ribeiro, J, P245

Ribes, A, P334

Ricardo, C P, P313

Ricci, E P, P300

Ricci, R, P179, P193

Richard, I, P388, P389

Richards, H, P267

Richards, V, P444

Richaud-Patin, Y, P171

Richter, J, OR025

Rico, S, P378

Riebesehl, N, P463

Riecken, K, P068, P456

Riess, O, P256

Rijal, P, P312

Rimann, N, P353

Rinaldi Ferreira, R, P459

Rincón, M Y, P287

Ringnalda, F, OR016

Rio, P, P027, P040, P260, OR012

Río, P, P043

Ripoll, C, P477

Rivat, C, P036

Rivis, S, P028, P361

Rizvanov, A, P138, P203, P204

Rizvanov, A A, P017, P081, P083, P088, P089, P139, P143, P146, P148, P152, P155, P169, P172, P207, P208, P218, P240, P243, P321, P365, P369, P377, P390, P391, P452, P476, P480 
Rizzi, N, P073

Rizzo, G, P439, P491

Roberts, M, OR010

Roberts, M L, P486

Rochat, C, P180

Rodino-Klapac, L, OR005

Rodionov, S A, P160

Rodríguez, S, P273, P302

Rodrigues, M, P381

Rodriguez-Madoz, J R, P273

Rodriguez-Perales, S, P260

Roelvink, P, P375

Rogozin, A A, P207

Roig-Merino, A, P122, P427

Román-Rodríguez, F J, P043

Roman-Rodriguez, F J, P040, P260, OR012

Romanel, A, P262, P299

Romano, M, P482

Romito, M, P108, P250, P283

Ronchetti, M, P142, P216

Rönnblom, L, P048

Ronzitti, G, P200, P345, P383, P388

Rosario, A, P444

Rosario, R, P223

Rosell, C, P177

Rositzka, J, P108

Roska, B, INV071

Rossella, V, P361

Rossetti, F, P457

Rossi, C, INV002

Rossi, G, P179, P213, P396

Rossi, S, P182

Rostonic, E, P444

Roswell, J, P286

Roth, F, P392

Rothe, A M, OR011

Rothe, M, P039, OR025

Rottensteiner, H, P037

Rouanet, M, P061

Rouger, K, P109, P113, P348

Rouillon, J, P341

Roujeau, T, INV076

rowlston, S, OR023

Roy, C, P439, P491

Roy, M, OR018

Rozhina, E, P147

Rozhina, E V, P012, P018

Rubic-Schneider, T, P322

Rubin, H, P344

Rubio, A, OR029

Rudilosso, L, P041

Rudolph, C, P108

Ruggiero, E, P311

Ruiz, J, INV075

Russell, D, INV070

Rutkowski, J V, P373

Rutland, C S, P391

Ryu, S, P297

Saburina, I, P134

Sachdeva, M, P307

Sadykova, F R, P006

Safiullov, Z Z, P139
Sagaradze, G D, P118, P137, P145, P160, P371

Sah, D, P178, P185, P190

Sahin, E O, P347

Sahin, U, OR028

Sakata, A, P241

Salafutdinov, I I, P139, P152, P476

Salas, D, P462

Salerio, F, INV002

Salerio, F A, P053, P104

Salido, E, P273, P293

Salikhova, T I, P006, P016

Salvarani, N, P227

Samain, F, P264

Samara, E, P287

Samaranch, L, P438

Sampson, C, P436

Samuelsson, S, P251

Samulski, J, INV065

San Sebastian, W, P438

Sanatine, P, P388

Sanberg, P R, P165

Sánchez, R, P040

Sanchez-Dominguez, R, P031, P120, P284, OR012

Sanchez-Nieto, J M, P313

Sanchez-Pernaute, R, P177

Sanchez, R, P027

Sanlioglu, A D, P086

Sanlioglu, S, P295, P347, P356

Santana, M, P222

Santiago, Y, P267

Santilli, G, P268, P283, OR020

Santoleri, L, P032

Santos Franceschini, J, OR009

Santos, J, P288

Santos, S G, P210, P236

Sanvito, F, INV002

Sapunova, A, P368

Saraiva, J, P222

Sarcar, S, P247, P287

Saricam, M, P013

Sarnowska, A, P011

Sartirana, C, INV002, P170

Sastre, L, P052

Sattler, R, OR018

Savic, N, OR016

Sawyer, E, P026

Sawyer, Y K, P235

Sayaka Nagamoto, S N, P291

Sayarova, R, P321

Scala, S, P032, P053, P104, P170

Scanziani, E, P105, P157

Scaramuzza, S, INV002, P028

Scardellato, S, P487

Štrancar, A, P007, P410

Scarpato, M, P200

Schäfer, R, P108

Schüssler-Lenz, M, INV010, INV045

Schaefer, P M, P354

Schaffer, D, INV052

Schalij, M J, INV056

Schallmoser, K, P102
Schambach, A, P039, P124, P166, OR011, OR025

Scharenberg, A M, P244

Scharler, C, P102

Scheel, M, P190

Schefter, L E, P244

Schejtman, A, OR020

Schenkwein, D, P455, P470

Scherer, T, P353

Scherman, D, P142, P216, P468

Scherz, A, P197

Schiermeier, A, P311

Schiller, L T, P459

Schiroli, G, P103, P254, P261, P489

Schlager, R, P047

Schleder, C, P109

Schleef, M, P328, P461, P475

Schlegel, A, P353

Schluep, M, P246

Schmeer, M, P328, P461, P475

Schmidt, M, P049, P319, P442, OR012

Schmidt, P, P019, P437

Schmit, P F, OR009

Schmueck-Henneresse, M, OR027

Schneider, B, P272

Schneider, B L, P180

Schneider, S, P459

Schob, K, P444

Schorge, S, P193

Schott, J W, P036

Schu, M, P352

Schultes, B, P311

Schultz, M, P188

Schutgens, R, P026

Schwabbauer, M, P124

Schwable, J, P026

Schwamborn, J C, P131

Schwank, G, OR016

Schwartz, J D, OR012

Schwarze, L, P456

Scott, E, P226

Scott, E W, P419

Sealy, G, P142, P205

Sebastian, S, P460

Seda, J, P411

Seeliger, F, INV069

Seeliger, M W, OR006

Segovia, J C, P027, P031, P120, P140, P273, P284, P293, OR012

Seidl, M, OR001

Seifried, E, P026

Seimetz, D, P161

Seitzer, J, OR018

Sellier, P, P341, P485

Selvakumaran, J, P196

Semenyuk, O, P219

Sena-Esteves, M, P222

Seneca, S, P274

Sengooba, A, P344

Seo, E S, P128, P158

Sereno, J, P101, P211, P222

Sergi Sergi, L, P105

Serina-Secanechia, Y N, P041

Servais, L, P378 
Sevilla, J, P027, OR012

Sevin, C, INV076

Seye, A, P445

Seymour, A, P344

Seymour, L, P466

Seymour, L W, P483

Shafigullina, A K, P365, P369

Shahrabi, A, P316

Shaimardanova, A A, P240, P243, P377, P390

Shakhnazarov, N A, P087

Shalaeva, Y, P364, P368

Shankar, R, P461, P475

Shaporeva, K R, P003

Sharma, P, P223

Sharon, D, P214

Sharrack, B, INV078

Shaw, C, P309, OR018

Shaykhulova, S F, P015

Shearman, M S, P195, P440

Shell, R, OR005

Shen, S, P217

Shi, X, P357, P447

Shi, Y, P323

Shiau, A L, P064, P398

Shieh, P B, P188, P378

Shihabuddin, L S, P256, P438

shimada, Y, P335

Shin, J, P116, P153

Shin, O K, P186

Shin, S H, P128, P158

Shipulin, G A, P281

Shivak, D, P267

Shivakumar, R, P301

Shmarov, M, P452

Shoaebargh, S, P418

Shortall, C, P217

Shteyer, E, P351

Shulman, I A, P207

Shum, I, P245

Shumatbaeva, A, P364, P368

Shupletsova, V V, P155

Sia, K C, P235

Sidahmed, H, P121

Silva, T P, P101

Silvestri, L, P028

Simó-Riudalbas, L, P080

Simon-Sola, M, P054, P485

Simonelli, F, P181, P182

Simonetti, E, P457

Sinagra, G, P227

Sinden, J, P430

Singh, G, P077

Singh, K, P287

Singh, L N, P354

Singh, M, P065

Singh, T, P037

Sinha, C, P403, P405

Siotou, E, P034

Siraeva, Z Y, P003, P016

Sitarou, M, P250, P279

Sitjve, S, INV005

Sitthirit, K, P133

Sivonen, M, P132
Skibo, Y V, P050, P051

Skor, M, P251

Slack, J, P467

Slobodkina, E S, P469

Slyk, Z, P079

Smart, M JK, P420

Smith, A J, P219, P245

Smith, B, P378

Smith, E C, P188

Smith, J, P108

Smith, J GW, P226

Snapper, J, P187

Snieckus, C, P438

Snyder, N W, P354

So, S, P116, P153

Sobczyk, A, P477

Sofronova, S A, P480

Sogorb-Gonzalez, M, P187

Sohl, N, P189

Sokolov, M E, P139, P208

Sola, M LS, P343

Sola, R, P298

Solanes, P, P272

Soleimani, M, P062

Solovieva, S, P362, P363

Solovyeva, V V, P081, P083, P088, P089, P143, P240, P243, P377, P390

Solovyova, V V, P172

Soncourt, A, P069

Song, C J, P440

Song, D, P253

Song, D W, P282, OR017

Song, Z, P353

Sorg, T, P264

Sourd, C, P252

Spada, A RL, P183

Spielmann, H, P124

Spinelli, A, P315

Spinozzi, G, P041, P332, OR008

Sproul, S, P267, P338

Sproule, D, P188, P395, P404, P411

Sproule, D M, P409, OR005

Sramek, M, P061

Srikanthan, M, P228

Srisook, P, P133

Srivastava, D, INV059

Srtrings-ufombah, V, P392

Stäble, S, P122

Stüber, T, P328

St. Martin, S J, P338

Staal, F J, OR011

Staal, F JT, P039

Stahlbom, A, P422

Stamatoyannopoulos, G, OR008

Stanek, L, P256

Stanek, L M, P438

Starostina, I G, P146, P240, P243, P377, P390

Stasi, L, P311

Steele-Stallard, H, P247

Stefanidakis, M, P251

Steger, K, P301

Steichen, C, P035, P381

Steiner, M, P231
Stephanou, C, P250

Stepniewski, J, P382

Stetkiewicz, P, P251

Stevens, M M, INV079

Stibolt, C, P191

Stijve, S, P005

Stok, M, P408

Stolzing, A, P115

Stone, L L, P165

Stoner, R, P294

Storch, S, P219

Storini, C, P482

Stornaiuolo, A, P314, OR004

Strange, C, P384

Strauss, K, P351

Strauss, K H, P333

Strebinger, D, P425

Strempel, N, P463

Stricker-Shaver, J, P256

Strina, D, P105, P157

Strings-Ufombah, V, P375

Stripecke, R, P107

Strullu, M, P027

Strunk, D, P102, P123

Studer, L, INV061

Stupnikova, T, P112

Su, B H, P398

Su, W, P443, P460, P466

$\mathrm{Su}, \mathrm{W} \mathrm{H}, \mathrm{P} 483$

Su, Y C, P064

Suarez, L, P233

Suh-Kim, H, P072, P163

Suhy, D, P375, P392

Sukhinich, K K, P202

Sukhorukov, G B, P155, P169

Sullivan, J, P438

Sultanova, E, P362

Summavielle, T, P210

Supraditaporn, K, P130

Surralles, J, P260, OR012

Susani, L, P105, P157

Suter, D, P306

Suter, D M, P305, P360, P425

Suzuki, T, P105

Swanson, X, INV006

Synnergren, J, P406

Syromiatnikova, V Y, P148

Syromyatnikova, V, P138, P203, P204

Szumilo, H, P079

Tabbaa, D, P251

Tabish, T, P045

Tagawa, M, P075

Tai, C, OR030

Tai, J A, P093

Tajnik Sbaizero, M, P007, P410

Takahashi, K, P392

Talmon, M, P171

Tan, W H, P110

Tanaka, M, P374

Tandre, K, P048

Tang, Y, P038

Tanniou, G, P485

Tarakanchikova, Y V, P169 
Tarasova, E Y, P168

Tardieu, M, INV076

Taromi, S, OR001

Tassara, M, P311

Tastanova, A, P322

Tasyurek, H M, P356

Tay, S, P135

Taylor, R, P346

Tazetdinova, L G, P089, P143, P172

Tedesco, F S, P119, P247

Teixidó, L, P334

Téjédor, G, P109

Temburni, S, P317

ten Bloemendaal, L, P357

Tenenbaum, L, P197

ter Brake, O, OR010

Testa, F, P182

Testasecca, A, P457

Thöny, B, P350, P353

Thalheimer, F B, P107

Themeli, M, P316

Theunissen, T W, OR013

Thoeny, B, OR016

Thomas, D, OR020

Thompson, J, P178, P185, P190

Thorne, L G, P489

Thrasher, A, INV025

Thrasher, A J, P036, P268, P283, P448, OR011, OR020

Thumann, G, P142, P205, P216, P468

Thumanu, K, P154

Tiberi, P, P181, P182

Tikhomirova, M V, P050, P051, P326

Timón, M, INV046

Timin, A S, P155

Timmers, A, P195

Timmers, A M, P440

Tinker, A, P226

Tintos-Hernandez, J A, P354

Tischlinger, K, P231

Tissot, L, P005, INV005

Titova, A A, P078, P087, P091, P365, P366, P369, P377

Titova, M A, P365, P369

Tiziano, E, OR004

Tkach, D, P317

Tkachuk, V A, P114, P137, P469

Tobalem, S, P205

Todosenko, N M, P155

Toivanen, P, P471

Toleikis, P M, P115

Tomás, J, P040

Tomé, S, P252, INV035

Tomczyk, M, P446

Tomelleri, A, P025

Topchu, Y A, P085

Torella, L, P273

Toriello, E, P181

Tornabene, P, P181

Torrance, V, P486

Torres-Ruiz, R, P260

Torres, R, P302

Torrisani, J, P069

Toumaniantz, G, P113
Towers, G J, P489

Townsend, A R, P312

Trabelsi, M, P351

Trapani, I, P181, P182

Trauchessec, M, P417

Traversari, C, P314, OR004

Trefely, S, P354

Treleaven, C M, P373

Trendell, J, P235

Trewman, S, P438

Trollet, C, P242, P375, P392

Trono, D, P080, P426, OR013, OR026, INV029

Truong, V, P444

Tsai, S Q, INV069

Tseng, S, OR030

Tsyplakov, D, P452

Tucci, F, P032

Tuckwell, N J, P201

Tulalamba, W, P274

Turaça, L T, P381

Turchiano, G, P108, P250, OR002

Türeci, Ö, OR028

Turelli, P, P426

Turkki, V, P453, P471

Turunen, H T, P195

Tutykhina, I, P452

Twigger, K, P144

Tyapkina, O V, P159

Tyurin-Kuzmin, P A, P118

U-Pratya, Y, P230

U-pratya, Y, P130, P133, P154

Uçkan-Çetinkaya, D, P030, P238

Ueffing, M, OR006

Ugalde, L, P027, P260

Ugolini, G S, OR029

Uhrig-Schmidt, S, P019

Ulfenborg, B, P406

Ulrich, P, P319, P322, P324

Ulum, B, P030

Unali, G, P489

Unzu, C, OR009

Ursu, S, P196

Usai, C, P233

Ustinov, K, P112

Vachey, G, P191, P270

Vader, P, P296

Vaggelas, A, P161

Vagin, V, OR018

Väistö, S, P471

Vakhrushev, I V, P202

Valeeva, A N, P081, P148

Valentinis, B, P314

Valeri, F, P171

Valeria, R, P028

Valero, I, P420

Vales, A, P273, P462

Valiullina, A, P321

Valiyakhmetova, A, P362

Valkama, A, P471

Vallès, A, P187

Vallanti, G, P457

Valle, M, P482
Vallianou, I, P428

Vallier, L, INV063

Valtolina, V, P314, OR004

Valton, J, P307, P317

Valtorta, M, P179, P213, P396

Van Brussels, K, P448

van Deventer, S, P187

van Dijk, T B, P279

van Eggermond, M, P039

van Eggermont, M, OR011

van Haasteren, J, P266

van Helden, R WJ, P355

van Heteren, J, OR018

van Roon, L, P039, OR011

van Royen, M E, P060

van Til, N P, P320

Van Wittenberghe, L, P341, P383

van Wittenberghe, L, P001, P485

van Wyk, M, OR015

van den Berg, J, P142

van den Bosch, T PP, P060

van der Burg, M, OR011

van der Hoeven, F, P122

van der Weiden, M M, P060

VanLith, C J, P258, P349, OR022

Vandamme, C, P471

Vandekerckhove, L, P049

VandenDriessche, T, P274, P287

Vandenberghe, L H, P337, P491, OR009

Vari, B, P123

Vasconcelos-Ferreira, A, P101

Vaubourg, C, P389

Vavassori, V, P254, P271

Venditti, C P, P336, P337, P491, INV043

Venditti, V P, P331

Venturoni, L, P336

Verbruggen, J, P009

Verhoeyen, E, P029, P107, P141, P300, INV014

Veron, P, P001

Vezzoli, M, INV002

Vezzoni, P, P105, P157

Viarengo, G, P032

Vidal, P, P383

Viemann, D, P117

Vihtelic, T S, P221

Vilas-Zornoza, A, P273

Viley, A, P301

Villa, A, P073, P105, P157, P254

Villanueva, M, P027

Villiger, L, OR016

Vilmont, V, P180

Vink, C, P473

Vink, C A, P436

Visigalli, I, INV002

Viswanathan, R, P257

Vlahovicek, K, P298

Vloemans, S, P039, OR011

Vo, T, P439

Vogel, A, OR028

Volk, H D, P102, OR027

Volkers, L, INV056

Voloshina, A, P368 
Volpin, M, OR008

von Kaisenberg, C, P124

Vorobev, V V, P006, P014

Vorobjev, I A, P143

Vrabec, K, P156

Vuillemenot, B, P223

Waddington, S N, P199, P380

Wagers, A, INV037

Wagner, D L, OR027

Wagner, J, P239

Wagner, R, P263

Walker, A, P269, P285

Wallace, D C, P354

Wallberg, M, P235

Walter, P, P216

Walther, J, P296

Walzer, K, OR028

Wang, C, OR018

Wang, C T, P398

Wang, H, P024, P034, P178

Wang, J, P251

Wang, R, P185

Wang, T, P257

Wang, W, P049, P185, P319, OR012

Wang, X, P185

Wang, Y S, P398

Wang, Z, OR018

Waselle, L, P005, INV005

Watano, R, P241

Watt, F M, INV040

Watts, C, P201

Weber, A, P035

Wechsler, T, P267, P338

Weißert, K, P167

Weisschuh, N, OR006

Weisser, M, OR020

Wellen, K E, P354

Wells, C, P188, OR005

Welstead, G G, P257

Wendering, D J, OR027

Wentworth, B M, P373

Westhaus, A, P448

White, C, P395

White, D, OR018

Whitehead, N, P352

Whyteside, G, P486

Wiedtke, E, P490

Wilbe, M, P048

Wilhelm, B, OR006

Willenbring, H, INV031

Williams, A, P307

Williams, J, P122

Williams, P M, P226

Wilson, C, P251

Wilson, C J, P257

Wimmer, M, P102

Wing, A, P309
Wissing, S, P463

Wissinger, B, OR006

Wolf, M, P102, P123

Wolf, P, OR001

Wolschek, M, P410

Wong, W Y, INV039

Wood, J A, P373

Wood, M, P196

Wood, S, P340

Woodall, M, P269

Woodford, D, P384

Wright, T L, P344

Wu, C C, P401

Wu, C L, P064, P398

Wu, E Q, P409

$\mathrm{Wu}, \mathrm{L}, \mathrm{P} 373$

Wu, N, P049

Wu, P, P064

Wynn, R, P340

Xiao, F, P165

Xiao, R, P337, OR009

Xiao, X, INV065

Xie, H, P165

Xie, J, OR018

Yakimkin, A, P148

Yakovlev, I A, P240, P367, P377, P390

Yamada, Y, INV050

Yamalitdinova, E I, P151

Yañez, J O, P486

Yañez, R, P031

Yañez, R M, P106, P140, OR012

Yáñez-Muñoz, R J, P196, P199, P380

Yang, G, P136

Yang, J, P294

Yanik, T, P030

Yannaki, E, P024, P034, P428

Yao, M, P373

Yarygin, K N, P202

Yarygina, N K, P202

Yasinovskiy, M, P112

Yasuda, M, P338

Ye, G, P440

Yen, M, P384

Yeom, S C, P282

Yergeshov, A A, P003, P014, P016

Ylä-Herttuala, S, P453, P455, P470, P471

Yliperttula, M, P073

Yonemitsu, Y, P374

Yoo, Y E, P465

Young, R M, P309

Ystaas, L R, P068

Yu-Taeger, L, P256

Yu, H S, OR017

Yu, X, P481
Yuan, C, P165

Yudkoff, C, P251

Yumi Kanegae, Y K, P291

Yurova, K A, P155

Zabaleta, N, P273, P462

Zacchigna, S, P262

Zahn, M, P319

Zakirova, E Y, P017, P081, P148, P149, P168, P391, P480

Zambelli, M, P032

Zambrowicz, B, OR018

Zammit, P S, P247

Zancan, S, INV002

Zanella, I, P262

Zanolini, D, P487

Zasso, I, P262

Žabar, R, P156

Zecca, M, P028

zeinali, S, P062

Zeinalova, A K, P377

Zeiser, R, OR001

Zenitilin, L, P007

Zentilin, L, P262, P298

Zérah, M, INV076

Zeynalova, A, P138, P203

Zgrundo, Z, P466

Zhang, G Q, INV056

Zhang, S, P346

Zheng, C, INV043

Zheng, K, OR018

Zhitko, A K, P017

Zhou, J, P178

Zhou, P, P185, P190

Zhou, R, P185

Zhu, C, OR019

Zhu, Y, P351

Zhuravleva, M, P321

Ziat, E, P379

Zierau, M, P115

Zimmer, V, P270

Zinchenko, S V, P078, P087, P091

Zinicola, M, OR020

Zinn, E, OR009

Zino, E, INV002

Zittersteijn, H A, P052

Zobor, D, OR006

Zoccolillo, M, P170

Zorbas, C, P027

Zörnig, I, P019

Zoughaib, M H, P003, P006, P014, P016

Zrenner, E, OR006

Zucchelli, S, P200, P487

Zurina, I, P134

Zuris, J A, P257

Zweegman, S, P316

Zweigerdt, R, P117

Zychowicz, M, P011 